\title{
The Stereoscopic Analog Trigger of the MAGIC Telescopes
}

\author{
F. Dazzi, T. Schweizer, G. Ceribella, D. Corti, A. Dettlaff, J. R. Garcia, D. Häfner, D. Herranz, M. López-Moya, \\ M. Mariotti, R. Maier, S. Metz, R. Mirzoyan, D. Nakajima, T. Saito, M. Shayduk, J. Sitarek, D. Strom, \\ M. Teshima, S. Tran, M. Will
}

\begin{abstract}
The current generation of ground-based Imaging Atmospheric Cherenkov Telescopes (IACTs) operate in the VeryHigh-Energy (VHE) domain from $\sim 100 \mathrm{GeV}$ to $\sim 100 \mathrm{TeV}$. They use electronic digital trigger systems to discern the Cherenkov light flashes emitted by Extensive Air Shower (EAS), from the overwhelming Light of the Night Sky (LoNS) background. Near the telescope energy threshold, the number of emitted Cherenkov photons by gamma-ray induced EAS is comparable to the fluctuations of the LoNS and the photon distribution at the Cherenkov-imaging camera plane becomes patchy. This results in a severe loss of effectiveness of the digital triggers. A stereoscopic analog trigger system has been developed for improving the detection capabilities of the Major Atmospheric Gamma-ray Imaging Cherenkov (MAGIC) telescopes at the lowest energies. It is based on the analog sum of the photosensor electrical signals. In this paper, the architectural design, technical performances, and configuration of this stereoscopic analog trigger, dubbed "SumTrigger-II", are described.
\end{abstract} ics.

Index Terms-Cherenkov telescopes, trigger systems, electron-

\section{INTRODUCTION}

$\mathbf{T}$ HE ground-based Imaging Atmospheric Cherenkov Telescopes (IACTs) detect gamma-rays from galactic and extragalactic sources in the Very-High-Energy (VHE) domain from $\sim 100 \mathrm{GeV}$ to $\sim 100 \mathrm{TeV}$ [1]. Cosmic- and gamma-rays generate Extensive Air Showers (EASs) when they enter the terrestrial atmosphere. These telescopes collect the Cherenkov light produced by relativistic charged particles in EASs, mostly by electrons, positrons, and muons. Dedicated off-line analyses of the EAS images ${ }^{1}$ recorded by IACTs allow identifying the nature of the primary particles, their arrival directions, and energies.

The development of EASs depends on the energy and nature of the primary particle. EASs initiated by low-energy primary gamma- or cosmic-rays develop faster and higher up in the

Manuscript received Month DD, 2021; revised Month DD, 2021; accepted Month DD, 2021. Date of publication Month DD, 2021; date of current version Month DD, 2021. This work was supported in part by the German BMBF and MPG, in part by the Italian INFN, and in part by the Spanish MINECO. (Francesco Dazzi and Thomas Schweizer are co-first authors.) (Corresponding authors: Francesco Dazzi; Thomas Schweizer; Giovanni Ceribella.)

F. Dazzi was with the National Institute for Nuclear Physics (INFN), I00044 Frascati, Italy. He is now with the National Institute for Astrophysics (INAF), I-00136 Rome, Italy (e-mail: francesco.dazzi.13@gmail.com).

T. Schweizer and G. Ceribella are with the Max-Planck-Institut für Physik, D-80805 München, Germany. (e-mail: tschweiz@mppmu.mpg.de; ceribell@mpp.mpg.de).

Digital Object Identifier XXXXXXXX

${ }^{1} \mathrm{~A} 2 \mathrm{D}$ angular projection of a 3D EAS on the Cherenkov-imaging camera, which is the telescope detector. terrestrial atmosphere, because the lower the initial energy of the primary particle, the less energy can be transferred to secondary shower particles. For example, the Cherenkov light produced by a gamma-ray initiated EAS with an energy lower than approximately $80 \mathrm{GeV}$, is mostly emitted in the upper layers of the terrestrial atmosphere above $10-12 \mathrm{~km}$. The Cherenkov light emission is less efficient at that altitude, due to the low air refraction index. Subsequently, a sizable fraction of Cherenkov light is absorbed along the path through the terrestrial atmosphere, further reducing the amount of light reaching the ground level.

Cherenkov light emitted at high altitude is strongly collimated along the trajectories of the charged EAS particles. This is due to the reduced Cherenkov emission angle at a lower index of refraction in air. EASs initiated by low-energy cosmic-rays are detected by IACTs as faint light flashes made of collimated light rays. In the case of EASs initiated by lowenergy gamma-rays $(\lesssim 80 \mathrm{GeV})$, these characteristics are even more pronounced because of the scarce photon statistics and their modest transversal development.

Finally, trajectories of particles in EASs are bent due to the interaction with the geomagnetic field [2]. EASs with the lowest particle energies are those developing highest in the atmosphere and therefore experiencing the greatest distortions due to the geometric field effect [3].

The images of EASs induced by low-energy primary gamma-rays, formed on the Cherenkov-imaging camera, are characterized by:

1) low photon density, close to the noise level (low Signalto-Noise $(\mathrm{S} / \mathrm{N})$ ratio);

2) photon distribution confined in a small region of the Cherenkov-imaging camera;

3) patchy photon distribution that complicates the reconstruction of the incoming direction of the primary particle;

4) low dispersion, of the order of nanoseconds, of the photons arrival time at the Cherenkov-imaging camera;

5) image center of gravity ${ }^{2}$ close (typically less than one degree) to the position of the gamma-ray source in the Cherenkov-imaging camera plane ;

6) image morphology distorted due to the geomagnetic field.

On the contrary, images of EASs induced by gamma-rays at higher energies are usually elliptical in shape, with most

\footnotetext{
${ }^{2}$ This is the weighted mean position of the charge.
} 


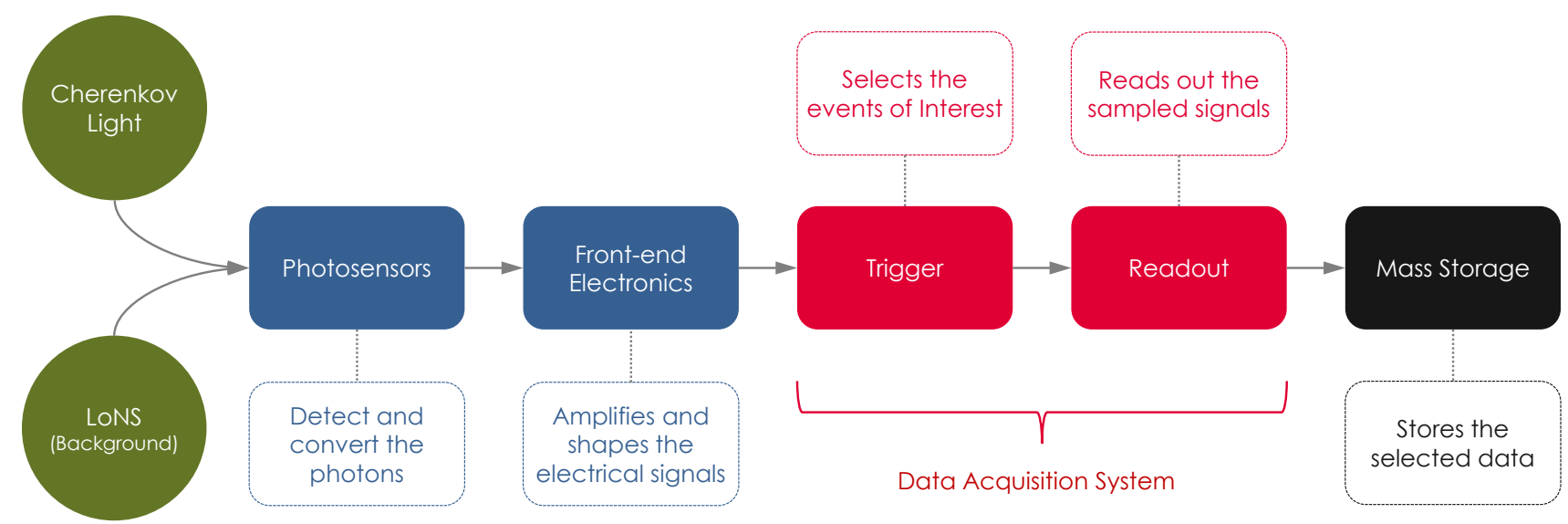

Figure 1. Working principle of an IACT. Green circles represent the radiation captured by an IACT. Filled blocks represent the main components of an IACT electronic chain, where the component functions are shown in unfilled blocks.

charge concentrated in the image portion corresponding to the maximum of the shower development.

These morphological characteristics of low-energy images, which reduce the performance of standard digital triggers, have motivated the construction of an analog trigger system, dubbed "Sum-Trigger-II". This trigger system has been installed in the Major Atmospheric Gamma-ray Imaging Cherenkov (MAGIC) telescopes. They are a stereoscopic system of two IACTs operating at the Observatorio del Roque de los Muchachos at the Canary Island of La Palma (Spain) [4]. Each telescope is composed of a tubular alt-azimuth mount, a parabolic $17 \mathrm{~m}$ diameter dish with active optics, a Cherenkov-imaging camera with 1039 PhotoMultiplier Tubes (PMTs), and a high speed Data Acquisition (DAQ) system [4]-[9].

\section{General Trigger Principles}

Fig. 1 shows the working principle of an IACT. Cherenkov photons are converted into electrical signals once they hit the photosensors of the Cherenkov-imaging camera. The trigger system processes these signals and generates a trigger when a correlation of the arrival time and amplitude is found in signals produced in neighboring photosensors. This process, which discerns the correlated signal generated by cosmic- and gamma-rays from the uncorrelated background noise, prevents from recording background events. The background noise is primarily due to the Light of the Night Sky (LoNS), which is mainly composed of integrated starlight, airglow, zodiacal light, and aurora [10].

In the case of an array of telescopes, the trigger system can be operated either in mono or stereo data-taking mode. In stereoscopic mode, the final trigger decision is taken if the event is triggered simultaneously by several telescopes. The constraint to require correlated events in multiple telescopes drastically reduces the accidental trigger rate due to the LoNS. It additionally rejects the background caused by low-energy muons. Consequently, the stereo data-taking mode significantly lowers the trigger thresholds without saturating the readout system.

Each MAGIC telescope is equipped with two independent trigger systems that are connected in parallel, as shown in Fig.
2. Standard observations are usually performed by using the digital trigger [11]. The analog trigger is the preferred mode for observations of sources, for which the bulk of the gamma emission is foreseen to happen at the very low energies as, for example, in pulsars. The simultaneous utilization of both trigger systems would increase the recorded data volume by about a factor of 2 and consequently the cost of operations and the data processing time, without providing a significant improvement of the scientific performance of the MAGIC telescopes.

Both trigger systems process the signals from the photosensors and generate single-telescope triggers. When the stereo data-taking mode is selected, the single-telescope triggers are transmitted to the stereo trigger system [12]. The stereo trigger is a coincidence logic accepting events that trigger both MAGIC telescopes in a narrow time window of $\approx 180 \mathrm{~ns}$.

In the digital trigger, the discriminators with a preset threshold convert photosensor analog signals into logic signals. These are then processed in a three-fold ${ }^{3}$ close-packed next neighbor coincidence logic for generating the single-telescope trigger. The three-fold next neighbor logic is well suited for triggering images left in the Cherenkov-imaging camera by cosmic- and gamma-rays with energies above approximately $80 \mathrm{GeV}$. At lower energies, the low photon density of the EAS images does not produce enough accumulated charge in the single photosensors to generate analog signals above the threshold of the digital trigger discriminators, namely a voltage equivalent to approximately four photoelectrons photoelectrons (phes).

In contrast to the digital trigger, the analog trigger system sums the analog signals of photosensors belonging to predefined Cherenkov-imaging camera patches (described in Section IV-A), whose size matches the angular extend of the images produced by cosmic- and gamma-rays below approximately $80 \mathrm{GeV}$. All signals within a camera patch are summed and processed by the analog trigger system, regardless of photon density and topology of the EAS images. The sum signal of

\footnotetext{
${ }^{3}$ The digital trigger can be configured to also process two-fold, four-fold, and five-fold close-packed patterns. The three-fold pattern is the standard MAGIC setting for stereo data-taking mode.
} 


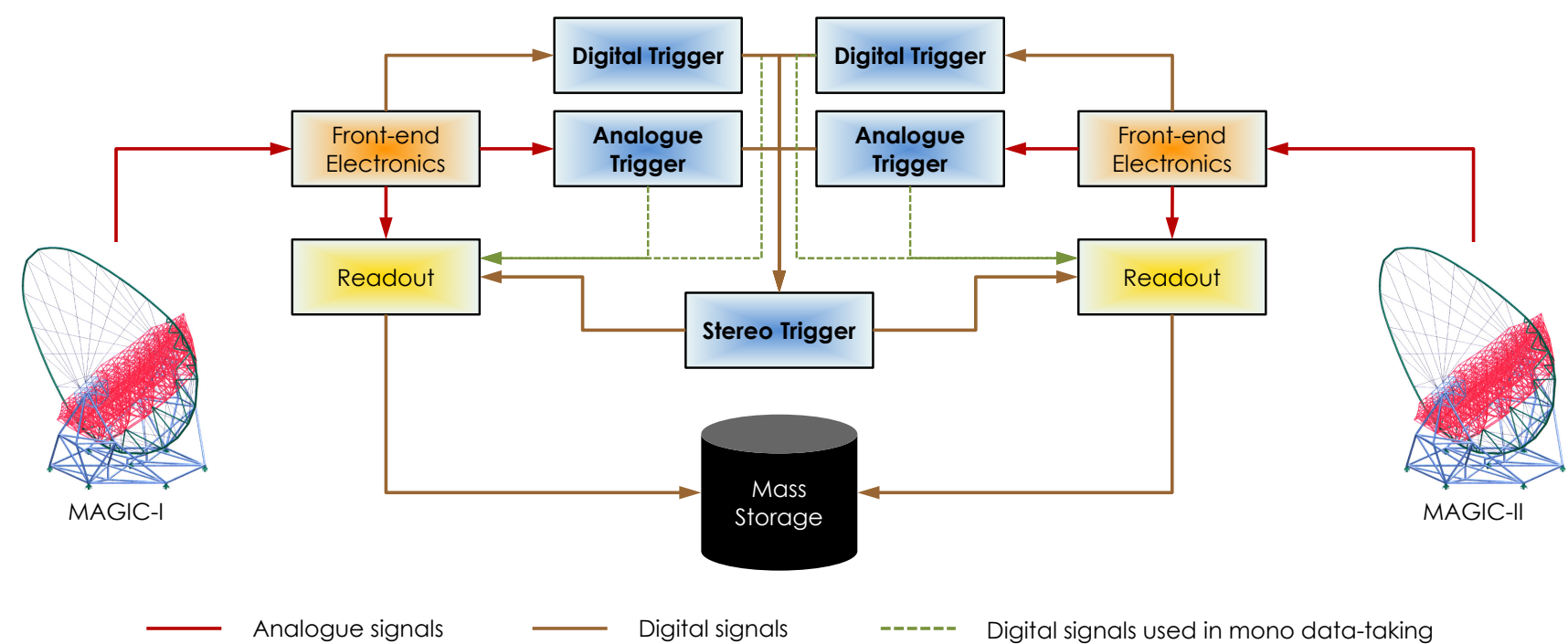

Figure 2. The context of the stereoscopic trigger systems of the MAGIC telescopes. Orange boxes represent the front-end electronics, which conditions the signals from the Cherenkov-imaging cameras for the trigger systems (blue boxes) and the readout system (yellow boxes). The readout system records in the storage mass (black box) only the signals that have passed the trigger criteria.

each camera patch is fed into a discriminator and the trigger is generated whenever the signal exceeds a preset threshold. All photosensors belonging to a camera patch contribute to the trigger decision, even if their signals are individually dominated by the fluctuations of the LoNS. Irregular and faint EAS images can generate triggers if many small signals from the EAS image are correlated in time and pile-up.

Considering that low-energy cosmic- and gamma-rays generate small, patchy, and irregular camera images, the principle to integrate over camera areas (i.e., camera patches) similar to the sizes of the images increases the $\mathrm{S} / \mathrm{N}$ ratio [13], [14]. The photosensor signals that are generated by an EAS are correlated and hence stack up linearly, while the noise components from different photosensors are nearly completely independent and hence stacks up in quadrature. Within a camera patch, the stacked up signal presents a significant improvement of the $\mathrm{S} / \mathrm{N}$ ratio. This makes the analog-sum trigger a high-performance system in the domain of few tens of $\mathrm{GeV}$ [15], [16], [19].

In 2008, the analog-sum trigger principle has been proven by the MAGIC collaboration, by the detection of the Crab Pulsar above $25 \mathrm{GeV}$ with a prototype system [20]. That system, dubbed "Sum-Trigger", showed remarkably good performance, as a very low-energy threshold, but it had some technological and operational limitations. It covered only a small fraction of the Cherenkov-imaging camera around its center, and its adjustment and fine-tuning had to be done manually. Moreover, the trigger was implemented only for monoscopic ${ }^{4}$ observations, which received high rates of background events at the lowest energies, strongly limiting the sensitivity of the MAGIC telescope.

The combination of the analog-sum trigger principle with the stereoscopic data-taking mode, enhances the sensitivity of the MAGIC telescopes, because stereoscopic observations

${ }^{4}$ The second MAGIC telescope was inaugurated on 2009. allow a strong background suppression. Therefore, a new stereoscopic analog trigger, dubbed "Sum-Trigger-II", has been developed by using the experience acquired during the construction and operation of the monoscopic prototype. The Sum-Trigger-II provides several improvements and new functions. For instance, it covers a larger part of the MAGIC Cherenkov-imaging camera area and the layout of the camera patches has been improved. The Sum-Trigger-II is fully adjustable (e.g., analog and digital delays, gains, thresholds) and controllable by an embedded small Linux computer. A semiautomatic procedure has been introduced for the calibration of the electronics, which has been installed in temperaturestabilized racks for maximizing the trigger response stability over time.

The Sum-Trigger-II has inspired the development of a similar trigger system for the Large Sized Telescopes (LSTs) [21], which are the largest telescopes of the coming Cherenkov Telescope Array Observatory (CTAO) operating in the energy domain $20 \mathrm{GeV}$ to $3 \mathrm{TeV}$ [22].

\section{MAin TRIGger REQUiREMEnTS}

The main Sum-Trigger-II requirements have been driven by scientific goals. Pulsars, Gamma Ray Bursts (GRBs), and distant Active Galactic Nuclei (AGNs) can provide fundamental information on extreme acceleration mechanisms and exotic physics, but their emissions have a cut-off at tens to hundred(s) of $\mathrm{GeV}$ [23], [24]. Thus, low-energy performance is essential for detecting and studying these objects.

Other important requirements, which significantly limit the solution space, are constraints associated with the installation of the Sum-Trigger-II into an already operative instrument, namely the MAGIC telescopes.

The most important performance requirements of the SumTrigger-II can be summarized as follows.

1) A trigger efficiency better than that of the digital trigger in the energy domain below $80 \mathrm{GeV}$. 
2) A trigger energy threshold, before off-line analysis, lower than $30 \mathrm{GeV}$ for a gamma source with a power law spectral index of -2.6 .

3) A trigger collection area, before off-line analysis, at least a factor of two greater than that of the digital trigger in the energy interval $30 \pm 10 \mathrm{GeV}$.

4) A PMT signal skew ${ }^{5}$ at the trigger summing stage lower than 250 ps Root Mean Square (RMS).

5) An amplitude equalization of the PMT signals better than $10 \%$.

6) A clipping mechanism able to counteract the effect of PMT afterpulsing.

7) A dispersion of the trigger response homogeneity over the Cherenkov-imaging camera aperture lower than 5\% for a dark Field of View (FoV) without bright stars.

8) A cross-talk in the analog path lower than $5 \%$.

9) A trigger linear range up to 100 phe, which is required for the calibration of the system.

10) An electronic noise at the discriminator level lower than 0.25 phe RMS for the standard PMTs gain.

11) A trigger output jitter lower than $1 \mathrm{~ns}$.

The most important operational requirements and constraints can be summarized as follows.

1) A smooth integration of the Sum-Trigger-II into the existing electronic chain of the MAGIC telescopes without the need for major hardware changes.

2) The installation of the Sum-Trigger-II in parallel to the digital trigger system.

3) A calibration process of the Sum-Trigger-II that can be executed during data-taking, while the parallel digital trigger is operative and using the interleaved calibration pulses $^{6}$, assuring no loss of observation time.

4) A trigger layout that covers more than $90 \%$ of the Cherenkov-imaging camera area covered by the digital trigger, in order to ensure the interoperability with the existing digital trigger and provide optimal performance during wobble-mode observations [25].

5) A trigger capable to operate in stereo data-taking mode.

6) A semi-automatic and user-friendly calibration process that can be performed by the telescope operators without the intervention of technical experts on the Sum-TriggerII hardware.

A fundamental design requirement is determined by the optimal camera patch size. Dedicated Monte Carlo studies have demonstrated that for the adopted PMT-based pixels with a FoV of 0.1 degrees, each camera patch should contain about 20 PMT-based pixels to obtain optimal performance in the energy domain around $25-30 \mathrm{GeV}$ [16]. Camera patches of smaller size have a higher probability to lose important components of the faint Cherenkov light from an EAS, while camera patches of larger size would increase the accidental trigger rates due to the LoNS.

\footnotetext{
${ }^{5}$ Relative time delay among electrical signals that are initially isochronous.

${ }^{6}$ During scientific observations, a calibration light flasher periodically illuminates the Cherenkov-imaging camera generating reference pulses, which interleave between pulses caused by the Cherenkov light.
}

\section{TRIGGER DESIGN}

\section{A. Layout of Camera Trigger Patches}

Systematic Monte Carlo studies have been performed with dedicated simulators [17], [18] to select the camera patch shape, size, and arrangement. The goal was to maximize the trigger efficiency, minimize the necessary overlap between the camera patches, and contain the electronics routing complication, without violating the dimensional constraints (e.g., available space and density of electric routing). Other aspects, such as cost, integration effort into the preexisting electronic chain, and the coverage of the digital trigger area, have also been considered during the analysis of these simulations. The outcome is a modular system that processes the signals generated in the inner part of the MAGIC Cherenkov-imaging camera, which is divided into three layers of patches, partly overlapping, as shown in Fig. 3.

Each camera trigger patch is composed of 19 PMT-based pixels $^{7}$ arranged to form a hexagonal shape with a FoV of almost 0.5 degrees. This patch size is optimized to obtain the best trigger efficiency for gamma-ray events in the energy domain around few tens of $\mathrm{GeV}$, whereas the hexagonal shape guarantees both a homogeneous and symmetrical overlap, and a central symmetry of the trigger area.

The camera trigger patches are arranged in the inner part of the Cherenkov-imaging PMT camera, because the images of the triggering low-energy EASs are formed within $\approx 1$ degree from the direction of the primary particle. This is caused by the fact that most of the faint Cherenkov light of low-energy EASs is emitted at the height of $10-12 \mathrm{~km}$ a.s.l and have an impact parameters up to $120 \mathrm{~m}$. Moreover, the extension of the Sum-Trigger-II to the whole Cherenkov-imaging camera has a low benefit-cost ratio, because it would require a redesign of most of the MAGIC data acquisition system and the stop of MAGIC telescopes operations.

The placement of the camera trigger patches is selected to maintain a circular symmetry, in which each patch is surrounded by six patches, equally placed every 60 degrees. This arrangement generates three superposed layers of patches that are indicated in Fig. 3a respectively with red, blue, and green. The obtained overlap is the minimal overlap that assures a homogeneous trigger response over the Cherenkov-imaging camera [19]. The homogeneity of the trigger response obtained during observations of an extragalactic field is shown in Fig. 4.

The key layout design specifications of the Sum-Trigger-II are summarized in Table I.

\section{B. Trigger Functional Architecture}

The Sum-Trigger-II is a modular system that independently processes the analog signals of each of the 55 camera trigger patches. The Sum-Trigger-II functional block diagram, limited to a single camera trigger patch, is illustrated in Fig. 5. The first two orange blocks shown in Fig. 5 represent the adjustment of the delays and gains of the PMT signals belonging

\footnotetext{
${ }^{7}$ Except for 12 outer camera trigger patches that contain 4 blind pixels each. In these camera trigger patches the sum is limited to 15 signals.
} 


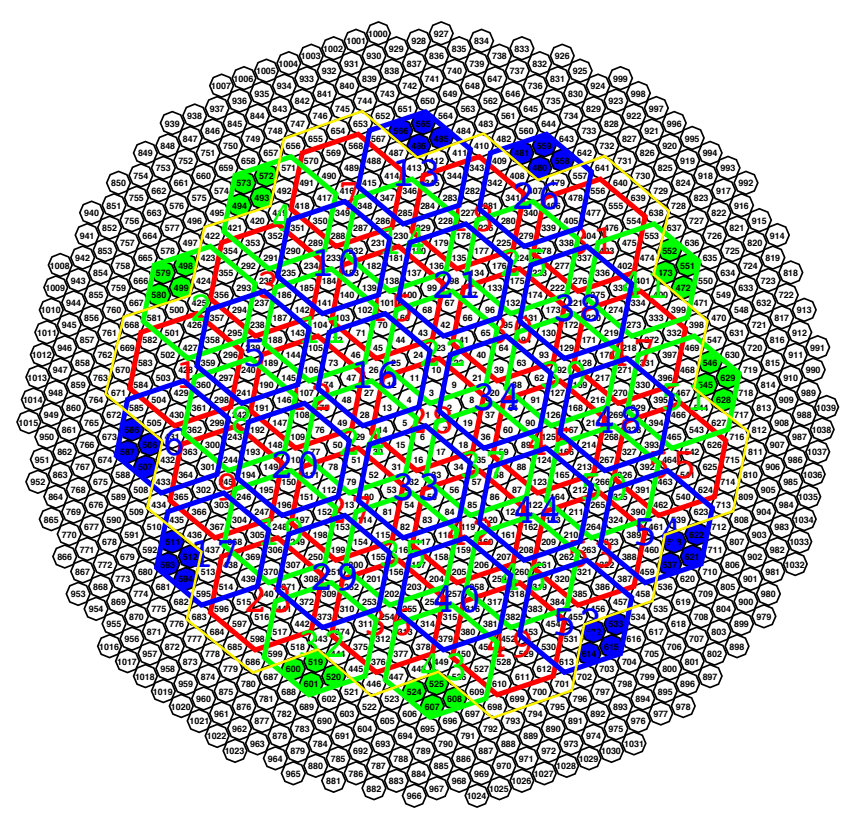

(a) Layers of camera trigger patches

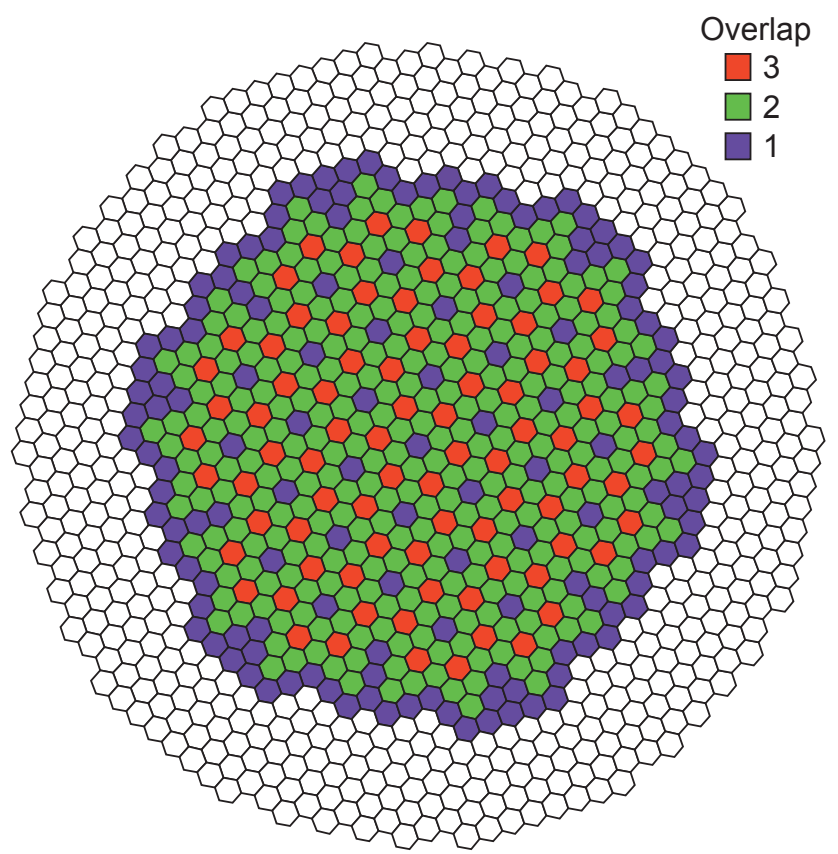

(b) Overlap density of the camera trigger patches

Figure 3. Illustration of the trigger area within a MAGIC Cherenkov-imaging camera overseen by the Sum-Trigger-II, where each small hexagon represents a PMT-based pixel [19]. (a) Arrangement of the camera trigger patches, which are distributed over three layers shown respectively with red, blue, and green color. Two out of these three layers, namely the blue and the green ones, have the same shape, but a different rotation around the Cherenkov-imaging camera center. The PMT-based pixels with a solid color fill are left out of the trigger decision to fill as best as possible the same trigger area as the digital trigger (yellow contour). (b) Density of the overlap between the camera trigger patches, where the colors indicate the number of patches to which each PMTbased pixel belongs.

to a camera trigger patch. These functions are important for obtaining an accurate and narrow signal pile-up, which is essential for good trigger effectiveness and a homogeneous trigger response over the entire camera trigger area. These functions are realized thanks to continuously variable analog

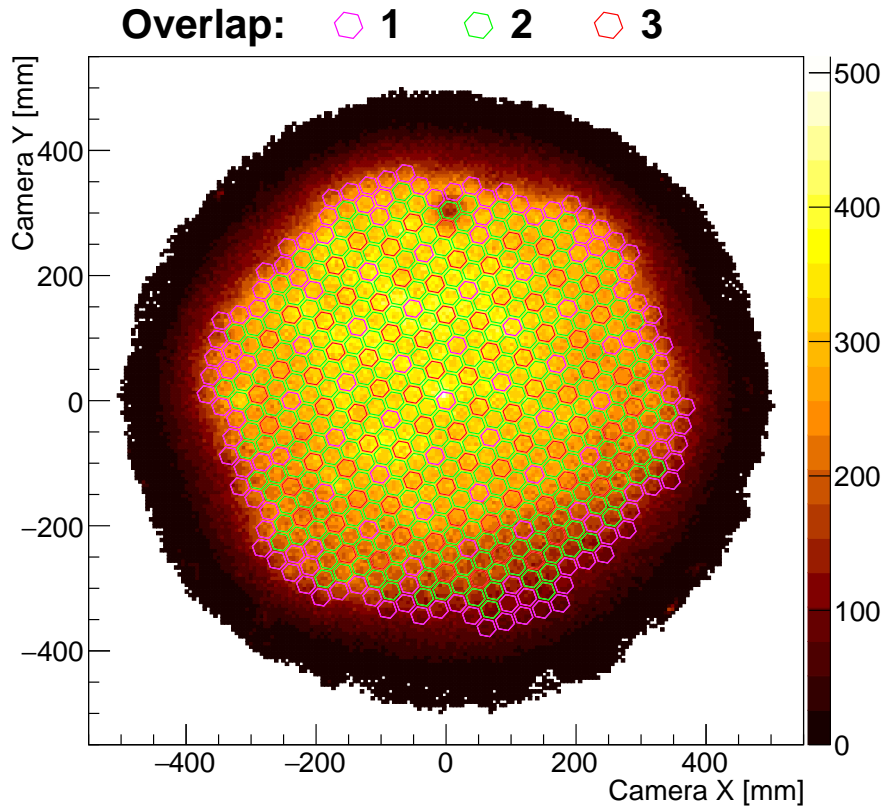

Figure 4. Distribution of the center of gravity of the images surviving the off-line image cleaning analysis in extragalactic field. The distribution is obtained by superimposing observations of the same source $(\mathrm{B} 0218+357)$ performed in different periods and with different wobble-mode configurations. This superimposition ensures a more symmetrical coverage of the trigger area. The overlaid hexagonal grid shows for each PMT-based pixel in Sum-TriggerII trigger area to how many camera trigger patches it contributes.

Table I

KEY LAYOUT DESIGN SPECIFICATIONS OF THE SUM-TRIGGER-II

\begin{tabular}{|l|c|}
\hline \multicolumn{2}{|c|}{ LAYOUT DESIGN SPECIFICATIONS } \\
\hline Name & Value \\
\hline PMT-based pixels in the Sum-Trigger-II area & 529 \\
Number of camera trigger patches & 55 \\
Shape of a camera trigger patch & Hexagonal \\
PMT-based pixels in each inner patch & 19 \\
PMT-based pixels in each outer patch & 15 or 19 \\
Electronic channels after splitting & 997 \\
Coverage of digital trigger area & $96.5 \%$ \\
\hline
\end{tabular}

signal delay lines and programmable attenuators.

PMTs suffer from large random noise pulses called "afterpulses". These are large spurious signals that appear sometime after the main pulse of a PMT. They are caused by the release and backward-accelerated ionized residual atoms and molecules, sitting on the surface of the first diode. These bombard the photocathode, thus producing large noise pulses [27]. The average after-pulse probability of the MAGIC telescope PMTs is about $10^{-3}$ per initial phe, therefore after-pulses are an important source of noise in an analog trigger system, as demonstrated by dedicated studies [15].

After-pulses from different PMTs can have large amplitudes up to $\approx 20$ phe, but they are not correlated in time, thus making it unlikely that two or more after-pulses can pile-up in the following summing stage. But even a single large-amplitude after-pulse from one PMT can contribute strongly to the sum of the 19 analog signals belonging to the same camera trigger 


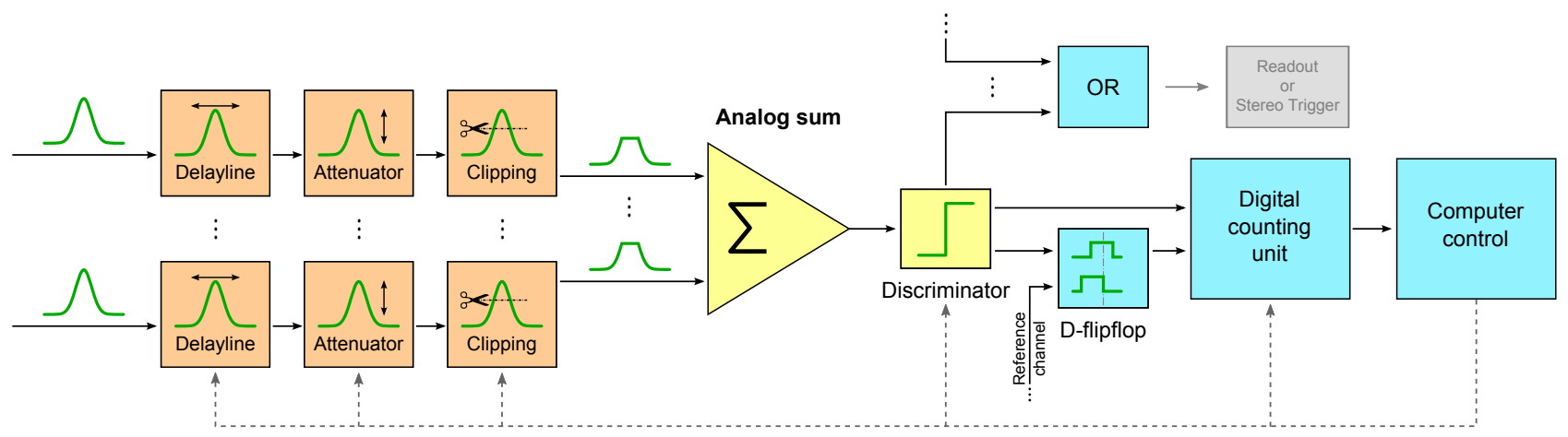

Figure 5. The functional blocks diagram of the Sum-Trigger-II, adapted from [19], [26]. The grey block does not belong to the Sum-Trigger-II and it depicts the possible destinations of the Sum-Trigger-II output (single-telescope trigger). In mono data-taking mode this output is transmitted to the readout system, while in stereo data-taking mode it is transmitted to the stereo trigger system.

patch, thus producing a trigger. Triggers due to after-pulses are unwanted and they can be minimized by cutting or clipping the PMT signals above a certain amplitude before the summing stage, as shown in the third orange block of Fig. 5.

The clipping function is a simple and powerful solution for rejecting after-pulses and preventing the generation of this type of fake triggers, which are dominated by the signal in a single PMT. The lower the clipping level, the better is the rejection of fake triggers due to after-pulses. However, low clipping levels can cut part of the signal from cosmic- and gamma-rays. Intermediate clipping levels shall be selected for optimizing the Sum-Trigger-II performance.

In stereo data-taking mode, it is possible to apply softer clipping cuts, because of the probability that huge afterpulses occur in both telescopes simultaneously is reduced by two-three orders of magnitude. Potentially, the clipping cuts could be further limited if the new generation PMTs with significantly lower after-pulse probabilities could be adopted in the MAGIC telescopes [28].

The summing and discriminator stages, yellow blocks shown in Fig. 5, represent the core function of the SumTrigger-II, where isochronous signals are piled-up and detected. Since the photons at the maximum of a gammaray induced EAS have a time spread of around $2.5-3 \mathrm{~ns}$, the bandwidth of the Sum-Trigger-II analog electronics has been carefully adjusted such that the signal Full Width Half Maximum (FWHM) is $\approx 3 \mathrm{~ns}$ at the summing stage. The signal pile-up within this time window optimizes the $\mathrm{S} / \mathrm{N}$ ratio at the discriminator stage. In fact, the time window of $\approx 3 \mathrm{~ns}$ is sufficiently narrow to minimize the rate of fake triggers produced by the LoNS, and at the same time it is sufficiently wide to maximize the detection of Cherenkov photons from EASs.

The cyan blocks in Fig. 5 represent the digital functions of the Sum-Trigger-II. The "OR" block combines the triggers generated in single-camera trigger patches into a unique telescope trigger. The "Computer Control" block represents the control and monitoring function. Finally, the remaining cyan blocks represent the main functions used for the calibration of the trigger system.

\section{Trigger Physical Architecture}

The Sum-Trigger-II is composed of two twin analog trigger systems, which are respectively implemented into the two electronic chains of the MAGIC telescopes. The twin systems are deployed into two standard Rittal 7820.510 closed racks, which are placed inside the electronic room of the MAGIC telescopes control building.

Fig. 6a shows the arrangement of the main Sum-Trigger-II Printed Circuit Boards (PCBs) within a rack and their main connections. The analog PMT signals are transmitted to each trigger rack via 70 shielded and differential high speed FCI cables, $5 \mathrm{~m}$ long. These cables are connected to $189 \mathrm{U}^{8} \mathrm{PCBs}$ dubbed "Clip-boards", which are placed in the upper part of the rack. These boards perform the signal delay, amplitude, and clipping adjustments, as shown in Fig. 6b. Thanks to a large-size and passive backplane, dubbed "Sum-backplane", the conditioned signals are then distributed to $193 \mathrm{U}$ PCBs dubbed "Sum-boards", which are placed in the lower part of the rack. Each Sum-board receives the conditioned signals from up to three camera trigger patches. The conditioned signals belonging to each camera trigger patch are piled-up and digital patch triggers are independently generated whenever the summed patch signal amplitude exceeds its corresponding discriminator threshold. The 55 patch trigger signals are then transmitted to a $9 \mathrm{U}$ PCB dubbed "Astro-board", which is placed in the upper-right part of the rack, as shown in Fig. 6a. 55 programmable ON NB6L295M delay chips, mounted on the Astro-board, nullify the patch trigger skews, which are introduced along the routing from the Sum-boards to the input of the Astro-board Field Programmable Gate Array (FPGA) ALTERA Cyclone IV - EP4CE15. The accuracy of the patch trigger skew cancellation will determine the jitter of the singletelescope trigger. In the FPGA, the patch triggers are combined into a global OR, whose output is used as single-telescope trigger. This signal is used either to trigger the readout system if the telescope is in mono data-taking mode or to participate in the stereo trigger decision if both telescopes are operated in stereo.

\footnotetext{
${ }^{8}$ This unit indicates the height of a PCB vertically mounted in a 19-inch rack, as defined in the standard IEEE 1101.1.
} 

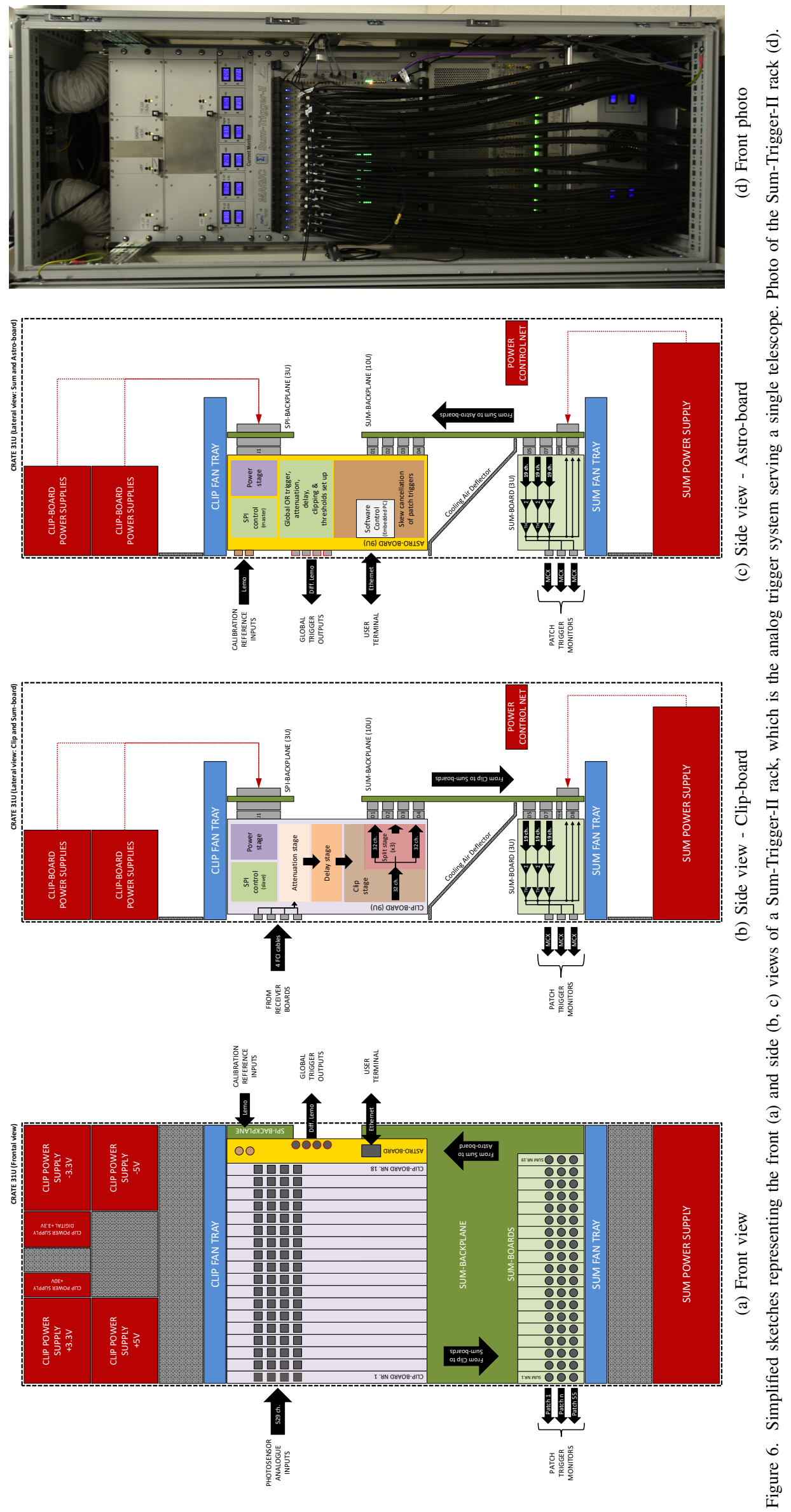
As shown in Fig. 6c, the Astro-board hosts a Linux embedded FOX Board G20 computer, where the control software of the Sum-Trigger-II runs. The control software is a multi-threaded C-program with a command-language interface, which is based on the MAGIC Integrated Readout program [29]. By default, the control software communicates with the MAGIC central control application [30] through an Ethernet connection. It can also be accessed from the operator terminal, for instance, for the execution of scripts that perform rate scans required during the calibration of the system. The control software sends commands to the Astro-board FPGA, which in turn controls and communicates with the ALTERA MAX-II Complex Programmable Logic Devices (CPLDs) of the Clip-boards through a Serial Peripheral Interface (SPI) and the Sum-boards. The Sum-Trigger-II commands are coded in 5 bytes and the whole set of commands has been hard-wired into the Astro-board FPGA. Specifically, this set of commands controls:

1) the analog delay lines in the Clip-boards;

2) the Clip-board attenuators;

3) the clipping thresholds;

4) the activation and deactivation of Clip-board channels;

5) the discriminator threshold in the Sum-boards;

6) the digital delay chips in the Astro-board;

7) the rate counters;

8) an independent coincidence trigger logic to monitor the stereo trigger rate.

The combination of a small embedded Linux system with an FPGA is powerful and sufficient to control the entire SumTrigger-II.

The configuration of the power supplies and the allocation of other auxiliary trigger functions to specific components are shown in the sketches of Fig. 6. A photo of a Sum-Trigger-II rack is shown in Fig. 6d, whereas photos of the single PCBs can be found in [31].

\section{Key Design Characteristics}

The Cherenkov light flash produced by a gamma-ray induced EAS typically lasts for 2-3 ns. The PMT signals generated by this Cherenkov light flash must retain a similar timing profile up to the summing stage. Therefore, the development of EASs in time constrains the bandwidth of the Sum-TriggerII electronics, at least up to the summing stage, where a signal distortion and/or broadening severely affects the effectiveness of the signals pile-up.

A typical PMT signal at the input of the Sum-Trigger-II appears like a pseudo-Gaussian pulse with a small falling tail [8]. The pulse rise time and FWHM are respectively $\approx 1.6 \mathrm{~ns}$ and $\approx 2.3 \mathrm{~ns}$. The discrete Fourier transform components of the pulse span within the range $10-700 \mathrm{MHz}$, with $90 \%$ of them contributing within the range $10-330 \mathrm{MHz}$. The pulse bandwidth $(-3 \mathrm{~dB})$ spans between $10-115 \mathrm{MHz}$.

The achievement of the required bandwidth has been hampered by the constraint to use long cables due to the layout of the MAGIC electronic room and the challenging signal routing along several electronics stages. The bandwidths of the SumTrigger-II components dealing with the PMT analog signals are reported in Table II. The bandwidth of the Clip-board is significantly lower than the others, so it is a critical board of the Sum-Trigger-II. The Clip-board bandwidth is a function of the transmission line parameters of its delay modules, so it depends on the applied delays. In the worst case, the high cut-off frequency appears at $400 \mathrm{MHz}$.

PMT signals can also be distorted by poor signal and power integrity. During the design development, the traces' impedance, the differential routing, and the PCB stack-ups have been analyzed and optimized to assure good signal integrity. Special care has been taken to limit both inductive and capacitive cross-talk, which introduces additional signal components that could generate fake triggers. Another critical aspect has been the decoupling of the analog circuitry from the digital one, mainly in the Sum-boards where inductive cross-talk from the digital transmissions could degrade the analog signal integrity or generate fake triggers. The Sumboard stack-up counts 14 layers, six for signals and eight for the ground and power planes. The PCB structure has been designed to obtain a differential impedance of $100 \Omega$ and a single-ended of $50 \Omega$. All of them are striplines disposed among ground layers, while the $\pm 5 \mathrm{~V}$ power planes are isolated at the middle of the board.

Furthermore, an incorrect signal arrival time at the summing stage affects the effectiveness of the signal pile-up. The skew between all ${ }^{9}$ analog signals is dominated by the contribution of the FCI cables and the chips mounted on the PCBs (see Table II). The contribution from the signal routing is negligible, because, in addition to programmable delay lines, meandering ${ }^{10}$ traces compensate the skew due to length or propagation delay mismatches. This routing strategy has been extensively used in the Sum-backplane, which is a $10 \mathrm{U}$ PCB with 20 layers, nine of which are reserved for signal routing. The lengths of the differential traces have been matched to the longest trace of $68.5 \mathrm{~cm}$. Another peculiarity of the Sumbackplane routing is that no vias have been included to avoid additional losses of the signal integrity. Fig. 7 shows the signal routing layers that contain the long meandering traces.

The material properties, as well as the physical characteristics of the PCBs, high-speed cables, and connectors have been carefully evaluated, tested, and selected. For example, the Sum-backplane utilizes the electrical insulator Nelco N400013. This material is an enhanced epoxy resin with a low dielectric constant of 3.4 , and has been engineered to provide both outstanding thermal and low signal-loss properties for high-speed applications. It also assures accurate impedance control. However, the manufacturing process has been very challenging and the required two units have been successfully produced only after several trials.

The key electronic characteristics of the entire Sum-TriggerII system are summarized in Table III. They maximize the integration of Cherenkov photons emitted by a gamma-ray induced EAS and provide a good $\mathrm{S} / \mathrm{N}$ ratio without violating the system requirements and the physical constraints.

\footnotetext{
${ }^{9}$ The skew within single camera trigger patches is much lower.

${ }^{10}$ The trace is not routed in a straightforward manner searching the minimal path, but a "zagging" method is used to match the longest trace.
} 
Table II

THE MAIN ELECTRONIC CHARACTERISTICS OF THE SUM-TRIGGER-II COMPONENTS

\begin{tabular}{|l|c|c|c|c|}
\hline \multicolumn{4}{|c|}{ PCBs AND CABLES CHARACTERISTICS } \\
\hline Name & Bandwidth & Cross-talk & RMS Noise & RMS Skew \\
\hline FCI cables & $\approx 550 \mathrm{MHz}$ & $<1.5 \%$ & $<0.03 \mathrm{phe}$ & $<250 \mathrm{ps}$ \\
Clip-boards & $\geq 400 \mathrm{MHz}^{*}$ & $<1 \%$ & $<0.03 \mathrm{phe}$ & Programmable \\
Sum-backplane & $\approx 625 \mathrm{MHz}$ & $<1.2 \%$ & Negligible & $<40 \mathrm{ps}$ \\
Sum-boards & $\approx 550 \mathrm{MHz}$ & $<1 \%$ & $<0.06 \mathrm{phe}$ & $<120 \mathrm{ps}$ \\
\hline
\end{tabular}

$(*)$ : measured with clipping disabled and no attenuation.

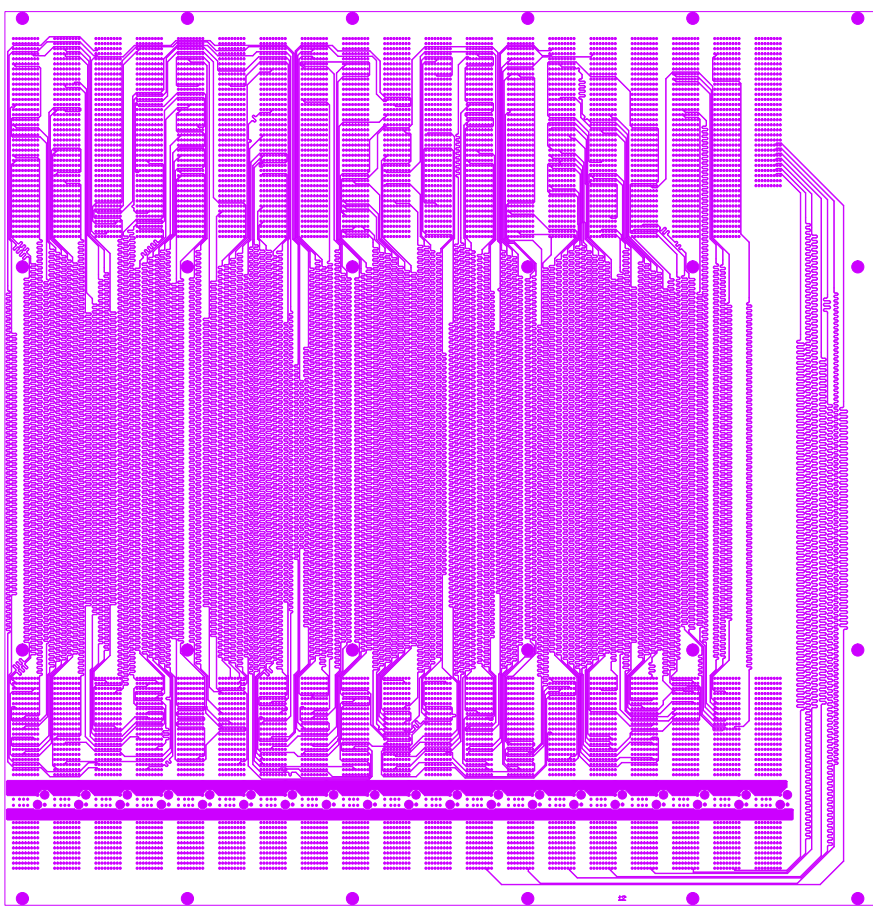

(a) Single signal routing layer

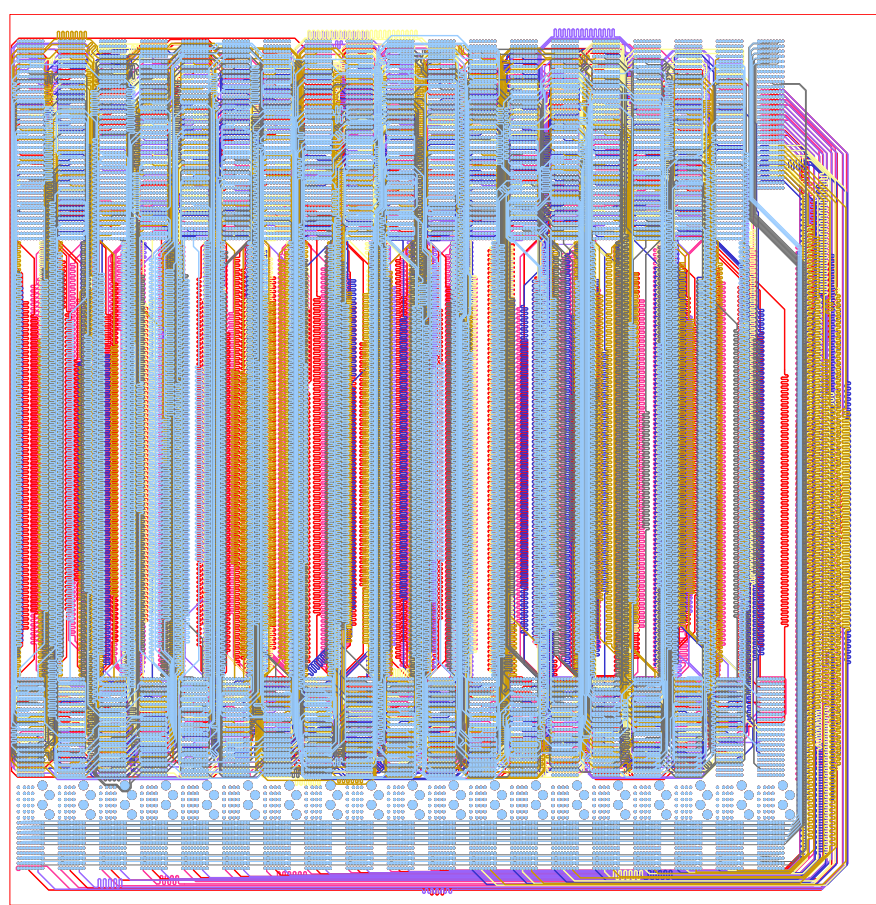

(b) All signal routing layers

Figure 7. Signal routing layers of the Sum-backplane, a large passive PCB measuring $44 \mathrm{~cm}$ in height and $42.8 \mathrm{~cm}$ in width. (a) One of the nine signal routing layer. (b) All overlapping signal routing layers.

Table III

THE MAIN ELECTRONIC CHARACTERISTICS OF THE SUM-TRIGGER-II

\begin{tabular}{|l|c|}
\hline \multicolumn{2}{|c|}{ SUM-TRIGGER-II CHARACTERISTICS } \\
\hline Parameter & Value \\
\hline Overall bandwidth & $>320 \mathrm{MHz}$ \\
Programmable analog signal delay range & $0-6.8 \mathrm{~ns}$ \\
Programmable analog signal attenuation range & $0--31.5 \mathrm{~dB}$ \\
Analog signal attenuation resolution & $0.5 \mathrm{~dB}$ \\
Pulse FWHM at summing stage (delay dependent) & $2.65-2.95 \mathrm{~ns}$ \\
Discriminator threshold stability & $<0.1 \mathrm{phe}$ \\
Overall cross-talk & $<3 \%$ \\
Overall electronic noise & $<0.13 \mathrm{phe}$ \\
Linear range maximum (without clipping) & $\sim 400 \mathrm{phe}$ \\
Trigger output jitter & $<0.5 \mathrm{~ns}$ \\
Total power consumption & $\sim 1800 \mathrm{~W}$ \\
\hline
\end{tabular}

\section{Trigger CAlibration}

Isochronous light flashes that homogeneously illuminate the MAGIC Cherenkov-imaging camera generate electrical signals that arrive at the Sum-Trigger-II with different delays and amplitudes. The different arrival times are mainly caused by the different signal transit times inside the PMTs and the different lengths of the optical fibers connecting the PMTs with the data acquisition system. The signal skews can attain huge values up to $6.5 \mathrm{~ns}$ over the entire Cherenkov-imaging camera. Similarly, the differences in the signal amplitudes are caused by the gain variations along the electronic chain from the PMTs down to the Sum-Trigger-II. The flat-field correction, applied to the MAGIC Cherenkov-imaging camera during standard observations, is not sufficient to achieve the required performance of the Sum-Trigger-II, because it equalizes the signal charge. A signal amplitude equalization is necessary for getting a homogeneous trigger response.

In each telescope, the trigger calibration is carried out 
by uniformly flashing the Cherenkov-imaging camera with a pulsed laser light source that generates homogeneous light flashes with a known intensity. The pulsed laser light source is a nanosecond light flasher, sitting in the dish center of each MAGIC telescope and pointing towards the Cherenkovimaging camera. The light intensity is set to produce electrical signals of the order of 50 phe in each PMT. The frequency of the light flashes is adjustable. During observations, the frequency is set to $25 \mathrm{~Hz}$ for generating interleaved calibration signals that do not increase the single-telescope trigger rate significantly. However, during technical operations the frequency can be set to $1 \mathrm{kHz}$ for a faster Sum-Trigger-II calibration.

The Sum-Trigger-II uses these light flashes for equalizing both the delays and the amplitudes of the PMT signals belonging to the camera trigger area. The calibration function reuses the same electronics adopted in the trigger mode, avoiding the implementation of expensive and complicated Analogto-Digital Converters (ADCs) and Time-to-Digital Converters (TDCs) in each channel. The calibration concept, based on scanning the signal amplitudes and delays, has been proven with a scaled prototype able to calibrate eight channels [26].

The calibration of the signal amplitudes and clipping levels is carried out via discriminator threshold scans, as shown in Fig. 8a. The rate of the discriminator output is recorded and plotted as a function of the discriminator threshold voltage. The collected data points are then fitted by a Sigmoid function and the signal amplitude is determined by computing the threshold value at the half-maximum of the Sigmoid function. The threshold scan is repeated for each signal of a camera trigger patch, one by one, by deactivating the other signals in the Clip-boards. Once all signal amplitudes are determined, the amplitudes are normalized to the average by changing the Clip-board attenuator settings.

The calibration of the signal delays is carried out by comparing the arrival time of all signals with respect to a reference one ${ }^{11}$, in a procedure called "delay scan" and shown in Fig. $8 \mathrm{~b}$. The analog signal to be calibrated in time is converted into a digital signal by the Sum-board discriminator, expressly set with a low threshold. This digital signal and the reference one are respectively transmitted to the input and clock ports of a flip-flop F-buffer of the Astro-board FPGA, where they are compared [32]. The flip-flop output reveals when there is a temporal overlap between the signal to be calibrated and the reference one, because it repeats the logic state of its input in correspondence with the clock timing, which is determined by the reference signal. The comparison is repeated by changing the delay of the signal to be calibrated, by spanning the whole second edge of the reference signal. The rate of the flip-flop output is recorded and plotted as a function of the applied signal delay, set in a Clip-board. The data points of a delay scan are fitted by a Sigmoid function, similarly to the curve fitting performed in the amplitude calibration. The calibrated delay is determined by computing the delay value at the halfmaximum of the Sigmoid function, with a timing resolution of

\footnotetext{
${ }^{11}$ The chosen reference signal is generated from a PMT installed outside the camera trigger area.
}

about $30 \mathrm{ps}$. This procedure is repeated for every analog signal by activating a single channel at a time within a camera trigger patch and deactivating the others.

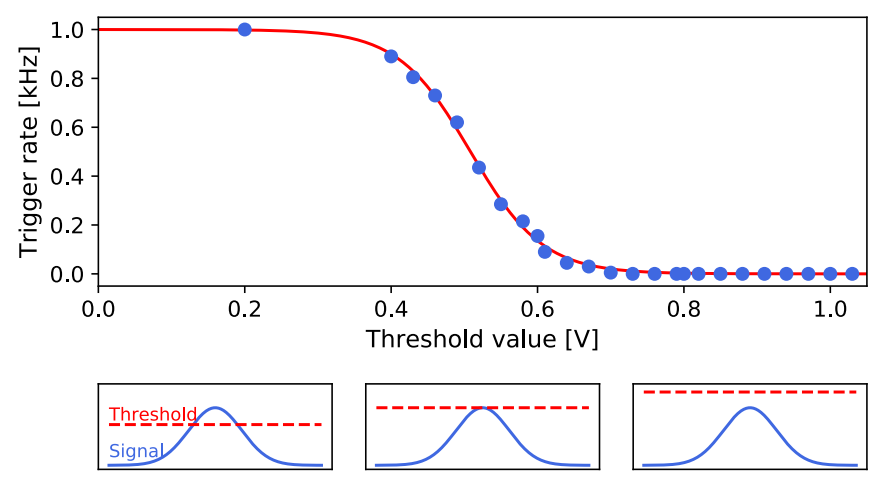

(a) Threshold Scan

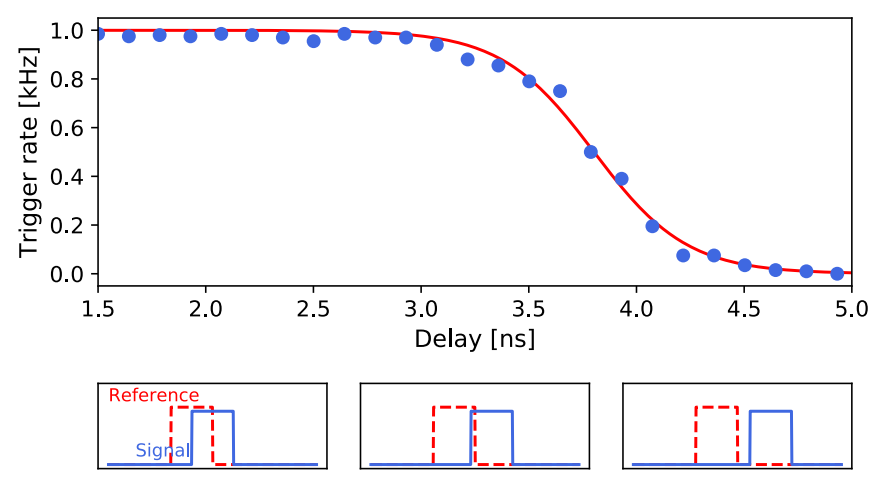

(b) Delay Scan

Figure 8. Typical rate scans obtained during the relative calibration of the signal amplitudes and delays. (a) The relation between the signal to be calibrated in amplitude and the reference threshold during the threshold scan. (b) The relation between the signal to be calibrated in time and the reference signal during the delay scan.

The calibration process is an iterative, semi-automatic procedure of amplitude and delay scans. The number of amplitude and delay scan cycles can vary, but usually two iterations are sufficient to achieve a calibration that satisfies the requirements. The outcome of a calibration process is shown in figure 9. The resulting delay and amplitude equalizations have a precision of about 100 ps RMS and 4\%, respectively.

\section{Trigger Configuration And Operational Mode}

Monte Carlo studies and tests have been carried out to determine the best Sum-Trigger-II configuration and operational mode. In particular, the optimal setting of the discriminator thresholds, single-telescope trigger rate, and clipping levels have been pursued for stereoscopic observations. The chosen standard operational mode maximizes the trigger performance by adapting in real-time the trigger configuration to the environmental and observing conditions. Any small variation of the atmospheric transmittance and/or the LoNS intensity in the telescope field of view requires the immediate adjustment of the discriminator thresholds. Moreover, the background is not uniform over the camera trigger area, so the optimal discriminator thresholds can change from one camera trigger 


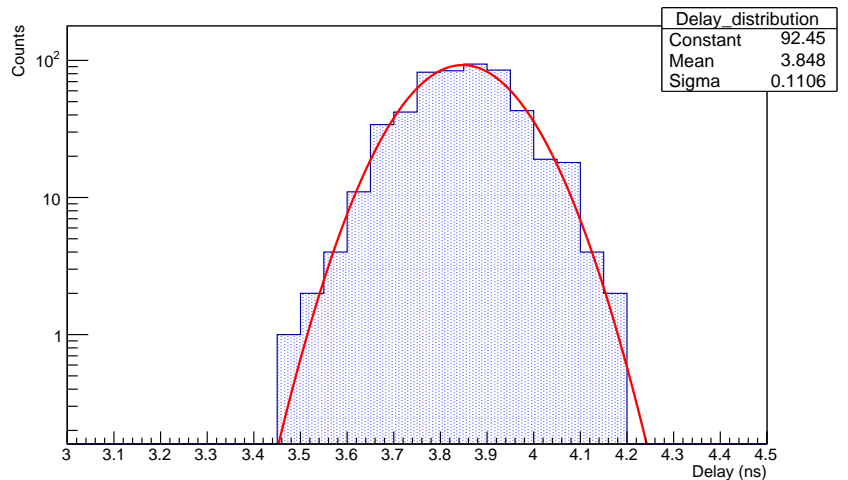

(a) Delay spread

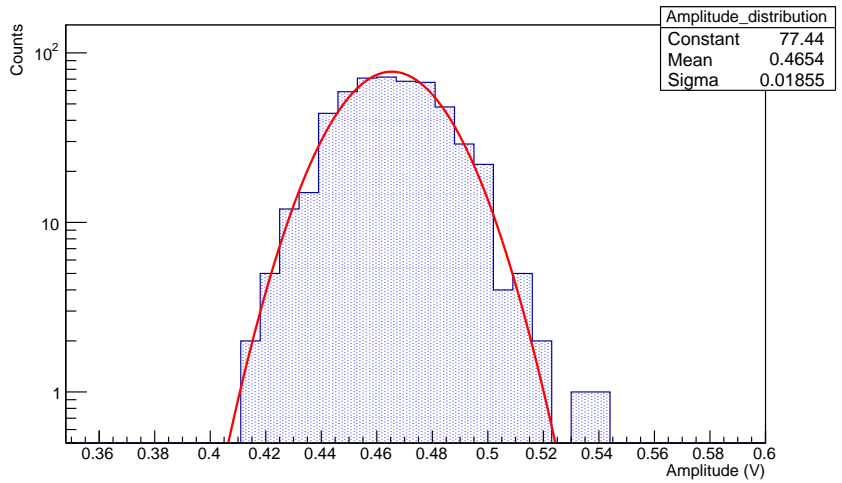

(b) Amplitude spread

Figure 9. Outcomes of the Sum-Trigger-II calibration process performed with the MAGIC-1 telescope after two iterations. (a) Distribution of the signal delays. (b) Distribution of the signal amplitudes.

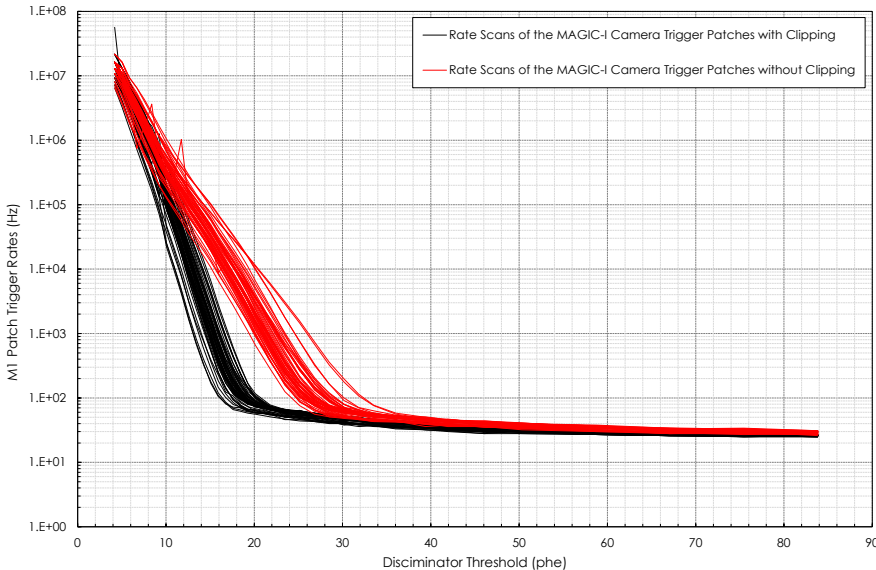

(a) MAGIC-I Camera Patch Trigger Rates

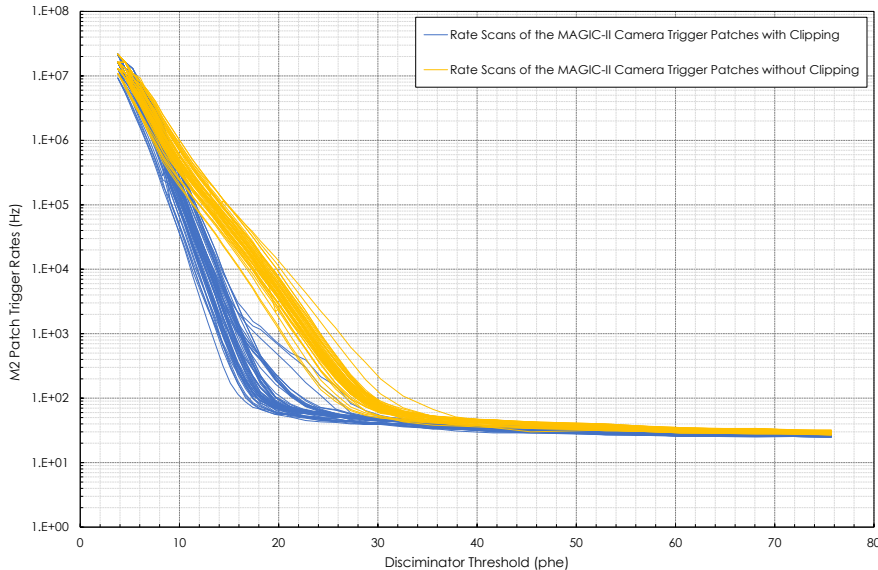

(b) MAGIC-II Camera Patch Trigger Rates

Figure 10. Rate scans of the camera trigger patches of the MAGIC telescopes during the observation of the galactic source Cygnus X-2 at zenith angle $32-35$ degrees, dust concentration $1.7 \mu \mathrm{g} / \mathrm{m}^{3}$, and atmospheric aerosol transmittance 0.93 (computed at $9 \mathrm{~km}$ above ground). Each curve is a rate scan of a camera trigger patch. (a) Rate scans of MAGIC-I camera trigger patches. (b) Rate scans of MAGIC-II camera trigger patches.

patch to another, as demonstrated by the rate scans of the camera trigger patches shown in Fig. 10.

During stereoscopic observations, the discriminator thresholds of the 55 camera trigger patches are continuously adjusted by two control loops acting within each other such that the individual camera trigger patches all have a similar patch trigger rate, while fixing the total single-telescope-trigger rate to $30 \mathrm{kHz}$. This control system function, called "Automatic Threshold Control", prevents saturating the readout system from exploding trigger rates due to stars and the other variable factors aforementioned. Since each camera trigger patch has different characteristics (e.g., PMT quantum efficiency) and is exposed to a different and variable background level, the applied discriminator thresholds are slightly different in absolute terms of phe. The inhomogeneity caused by applying different discriminator thresholds is mitigated by the image cleaning procedure during the off-line data analysis. The image cleaning procedure has a higher threshold and homogenizes the shower distribution over the Cherenkov-imaging camera. The Automatic Threshold Control minimizes the effect of the LoNS on the trigger response homogeneity and assures the maximally lowest possible trigger and analysis energy threshold without saturating the readout system.

The typical stereo trigger rate is about $500-600 \mathrm{~Hz}$, for galactic sources at a low zenith angle below 30 degrees and an atmospheric transmittance higher than 0.85. Most of these triggers are due to EASs, whereas approximately $160 \mathrm{~Hz}$ are accidental triggers due to the LoNS, which get eliminated in the off-line data analysis. The contribution of spurious stereo triggers due to after-pulses is negligible if the clipping levels are properly configured.

Simulations of the trigger efficiency, by varying the clipping levels, has revealed that a clipping setting in the range 610 phe is optimal. It reduces the rate of spurious singletelescope triggers due to after-pulses down to a level well below the EAS trigger rate, while limiting the losses of gammas. The simulation results have been verified with test stereo data, where the number of triggered EASs has been recorded as a function of the clipping level. The test data have confirmed that the maximum EAS trigger rate is obtained with 
a clipping level in the range $6.5-8.5$ phe, as shown in Fig. 11 . A clipping level of about 8 phe has been set in both MAGIC telescopes.

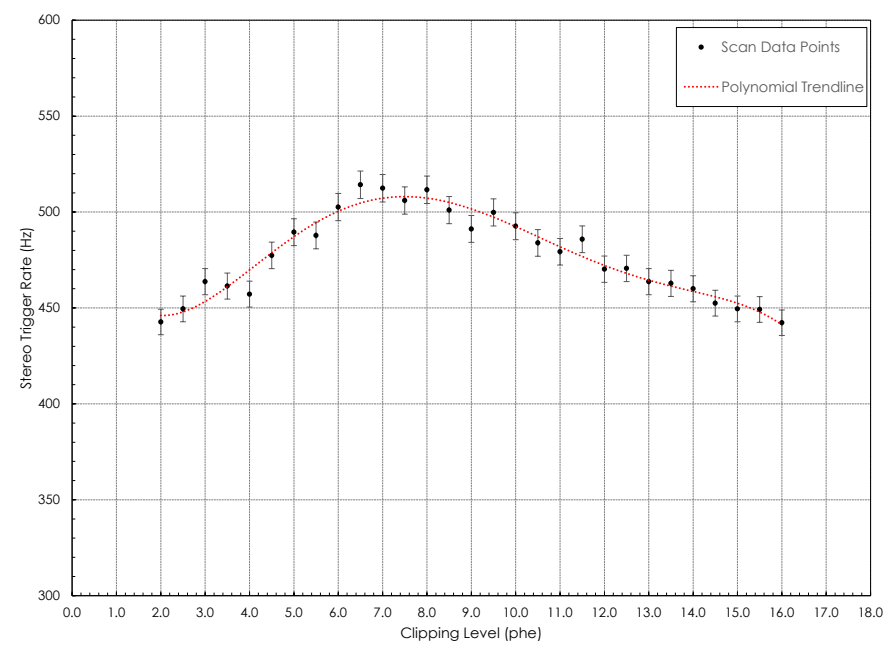

Figure 11. Stereo trigger rate as a function of the clipping level measured during the observation of the galactic source Cygnus X-2 at zenith angle $23-$ 31 degrees, atmospheric transmittance 0.93 , and dust concentration $1.7 \mu / \mathrm{m}^{3}$. The total stereo trigger rate is determined by the sum of the EAS trigger rate, constant accidental trigger rate, and after-pulse trigger rate. Given the singletelescope-trigger rate is fixed, the peak of the stereo trigger rate occurs when the clipping level is sufficiently high to minimize the loss of EAS triggers and sufficiently low to minimize the fraction of after-pulse triggers that contribute to the fixed single-telescope trigger rate.

\section{TRIGGeR PERFORMANCE}

Fig. 12 shows the single-telescope-trigger rate scans during the observation of a galactic source. MAGIC-I and MAGICII trigger rate scans are very similar. This assures that the Sum-Trigger-II systems installed in both telescopes are well integrated and inter-calibrated. These trigger rate scans also confirm what has been predicted in dedicated trigger simulations. An effective discriminator threshold for camera trigger patches of 19 PMT-based pixels is usually around 2-3 times the set clipping level ${ }^{12}$, which has been set to about 8 phe. Last, but not least, these trigger scans highlight the importance of the clipping function in enhancing the detection capability of low energy events. At the single-telescope working point, the average discriminator threshold is significantly lowered when clipping is applied.

The potential scientific performance of the Sum-TriggerII has been determined by simulating the system with the aforementioned configurations. 64 million gamma-ray events from a source with a power law spectral index of -2.6 and a maximum impact parameter of $400 \mathrm{~m}$ in respect to the center of the telescope array have been simulated in combination with a simulated LoNS flux of the order of $0.19 \mathrm{Ph} /\left(\mathrm{cm}^{2} \cdot \mathrm{sr} \cdot \mathrm{ns}\right)$, which produces an electronic noise in the MAGIC telescope Cherenkov-imaging cameras of $0.11 \mathrm{phe} / \mathrm{ns}$ and $0.12 \mathrm{phe} / \mathrm{ns}$ per PMT respectively. These simulations have been used to compute the trigger collection area (Fig. 13a) and energy

\footnotetext{
${ }^{12}$ This holds true only for the PMTs currently used in the MAGIC telescopes.
}

distribution (Fig. 13b), of which the peak denotes the expected energy threshold of the stereoscopic trigger system.

Fig. 13 shows the Sum-Trigger-II capability to trigger gamma-rays before any off-line software analysis. Fake triggers generated by the LoNS have been removed. In the case of real data, most of these fake triggers are removed by offline image cleaning algorithms, and any remaining ones are removed during the background subtraction process.

Below $80 \mathrm{GeV}$, the Sum-Trigger-II presents superior performance compared to the digital trigger. The resulting stereo Sum-Trigger-II energy threshold is significantly lower $(21 \mathrm{GeV})$ than that of the standard digital trigger $(41 \mathrm{GeV})$ and similar to the single telescope threshold of the prototype SumTrigger [15]. Around $40 \mathrm{GeV}$, where the digital trigger has its energy threshold, the Sum-Trigger-II capability to trigger is twice as large. At $30 \mathrm{GeV}$ the improvement increases to approximately a factor four, and at $20 \mathrm{GeV}$ is more than a factor six. Regarding the collection area, the performance of the Sum-Trigger-II is much better along the entire operative range below $80 \mathrm{GeV}$. At few tens of $\mathrm{GeV}$, the improvement is remarkable. At $20 \mathrm{GeV}$, for example, the Sum-Trigger-II collection area is already $\approx 9000 \mathrm{~m}^{2}$, an order of magnitude larger than the digital trigger collection area.

\section{CONCLUSION}

Since the beginning, the MAGIC telescopes has adopted a multi-level digital trigger system to reject accidental triggers due to the LoNS in real-time. This is very effective above $\approx 80 \mathrm{GeV}$, but it shows limitation at lower energies. Attractive physics questions, mainly related to Pulsars, distant AGNs, and GRBs, have stimulated the development of the Sum-TriggerII, which is the most effective system at these energies below approximately $80 \mathrm{GeV}$.

The Sum-Trigger-II has been installed and commissioned in 2014 and 2015 on both MAGIC telescopes, enabling stereo observations in the low-energy domain [32]. Since 2016, the system is used for special scientific projects focused on the low-energy domain. The good performance of Sum-TriggerII, derived from the observation of real sources, has confirmed the design expectations [33]-[35].

\section{ACKNOWLEDGMENT}

We want to thank the Instituto de Astrofísica de Canarias for the excellent working conditions at the Observatorio del Roque de los Muchachos in La Palma.

We want to thank the electronics workshop of the MaxPlanck-Institute for Physics in Munich for the technical support. In particular we want to thank D. Fink, J. Hose, P. Grundner, and M. Reitmeier, who contributed to the design, production, and testing of the Clip-boards, delay modules, rack, and cooling system.

We want to thank the electronics workshop of the Istituto Nazionale di Fisica Nucleare in Padua for the technical support. In particular we want to thank M. Bettini and P. G. Zatti, who contributed to the development of the PCB layouts of the Sum-board and Sum-backplane. 


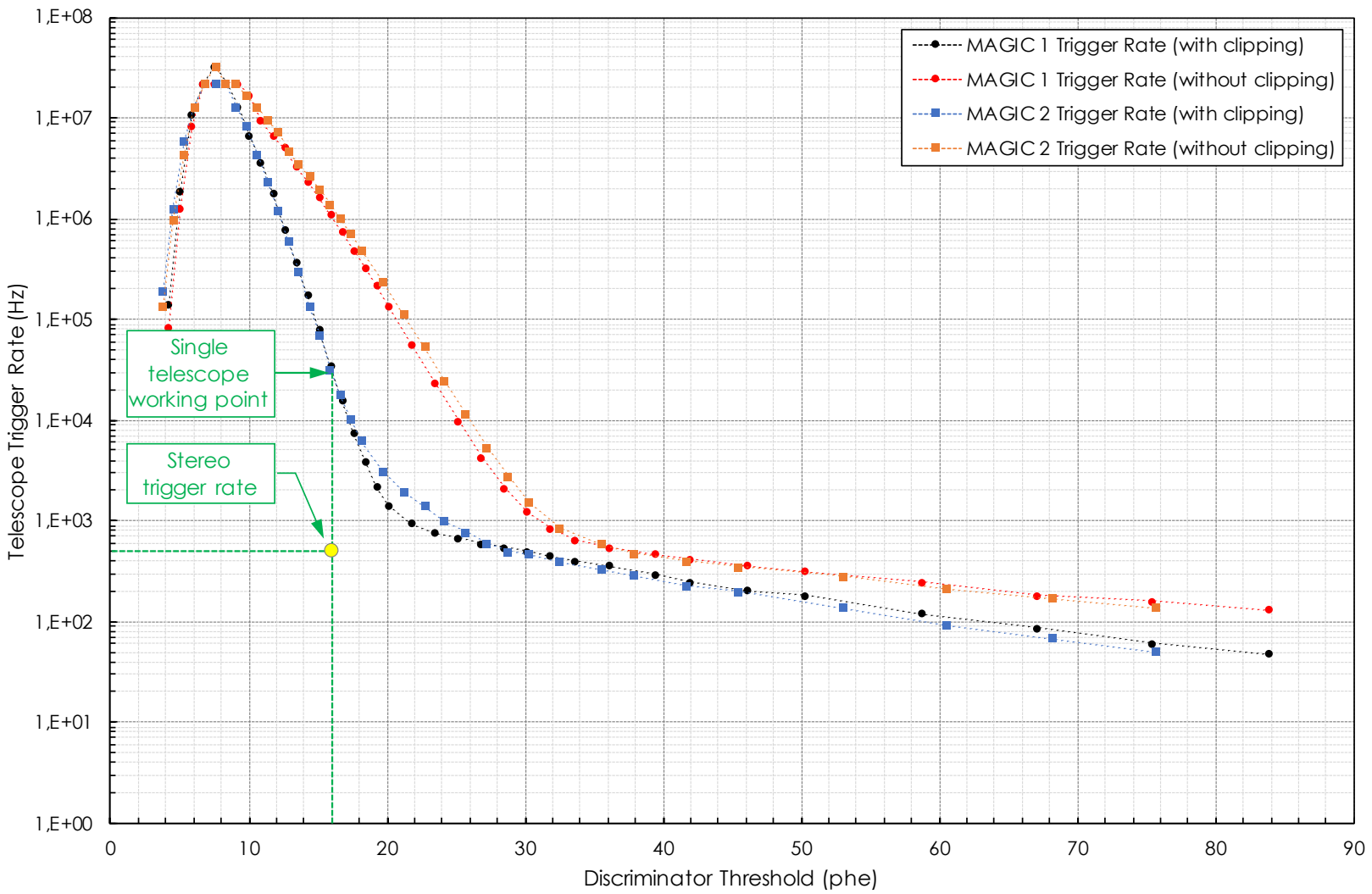

Figure 12. Single-telescope-trigger rate scans during the observation of the galactic source Cygnus X-2 at zenith angle $32-35$ degrees, atmospheric aerosol transmittance 0.93 , and dust concentration $1.7 \mu \mathrm{g} / \mathrm{m}^{3}$. Below 8 phe the single-telescope trigger rate drops because the 55 patch trigger signals become so frequent that they start overlapping and their subsequent "OR" changes logic state less frequently.

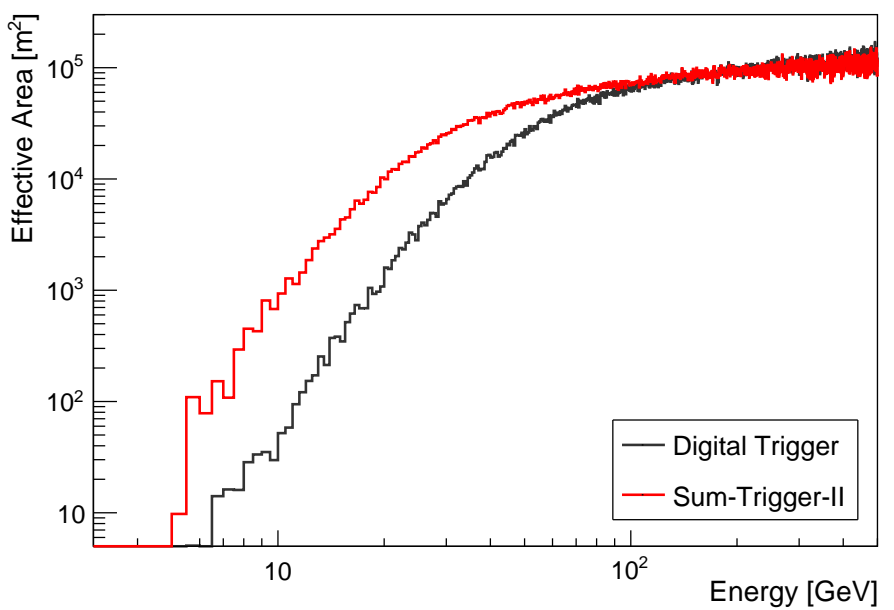

(a) Stereo collection area

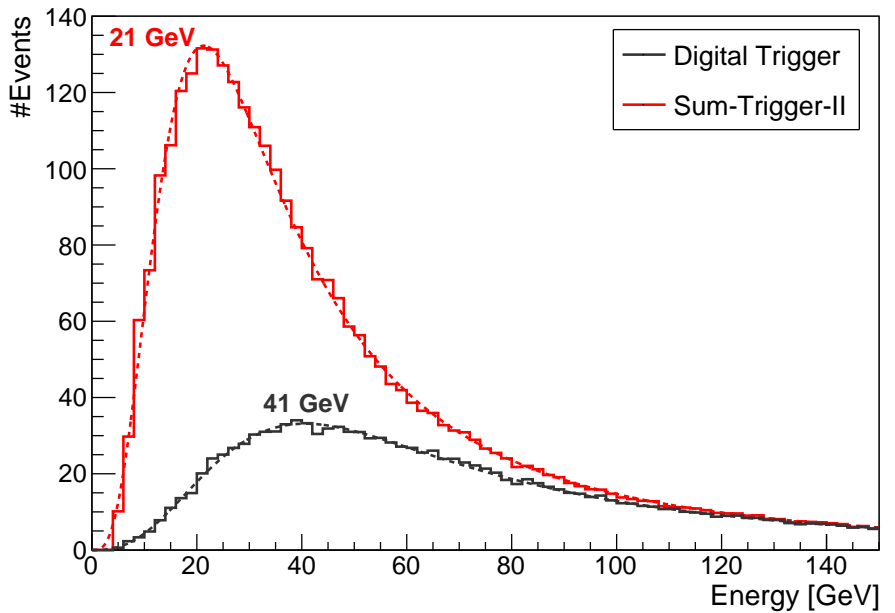

(b) Stereo energy distribution

Figure 13. Trigger capability to select gamma-rays from a source with a power law spectral index of -2.6 . (a) Collection area for the MAGIC digital trigger (black) and the Sum-Trigger-II (red). (b) Number of stereo events close to the threshold (peak of the distribution) for the MAGIC digital trigger (black) and the Sum-Trigger-II (red) configurations.

We want to thank D. Mazin and R. Paoletti, who shared their experience on the MAGIC Integrated Readout program and supported the development of the Sum-Trigger-II control system.

We want to thank the members of the Sum-Trigger-II review committee, O. Blanch, R. Zanin, and L. A. Tejedor, who provided an accurate assessment and valuable feedback.
We want to thank the members of the local MAGIC crew, J. H. Llorente and E. Colombo, for their logistics support.

Finally, we want to thank the MAGIC collaboration for allowing and supporting this initiative.

\section{REFERENCES}

[1] T. C. Weeks et al., Observation of $\mathrm{TeV}$ Gamma Rays from the Crab Nebula Using the Atmospheric Čerenkov Imaging Technique, 
in The Astrophysical Journal, Volume 342, p. 379, July 1989, doi: $10.1086 / 167599$.

[2] R. De Los Reyes et al., Low Energy $\gamma$-ray Detection $(\mathrm{E} \leq 30 \mathrm{GeV})$ : Effect of Earth's Magnetic Field and a Novel Trigger Technique, in International Journal of Modern Physics A, Volume 20, No. 29, pp. 7006-7008, November 2005, doi: 10.1142/S0217751X05030697.

[3] S.C. Commichau et al., Monte Carlo studies of geomagnetic field effects on the imaging air Cherenkov technique for the MAGIC telescope site, in Nuclear Instruments and Methods in Physics Research Section A: Accelerators, Spectrometers, Detectors and Associated Equipment, Volume 595, Issue 3, pp. 572-586, October 2008, doi: 10.1016/j.nima.2008.07.144.

[4] J. Aleksić et al., The major upgrade of the MAGIC telescopes, Part I: The hardware improvements and the commissioning of the system, in Astroparticle Physics, Vol. 72, pp. 61-75, January 2016, doi: 10.1016/j.astropartphys.2015.04.004.

[5] J. Cortina et al., Technical Performance of the MAGIC Telescopes, Proceeding of 31st International Cosmic Ray Conference (ICRC 2009), Lodz (Poland), 7-15 July 2009, arXiv:0907.1211.

[6] T. Bretz et al., The drive system of the Major Atmospheric Gamma-ray Imaging Cherenkov Telescope, in Astroparticle Physics, Volume 31, Issue 2, pp. 92-101, March 2009, doi:10.1016/j.astropartphys.2008.12.001.

[7] M. Doro et al., The reflective surface of the MAGIC telescope, in Nuclear Instruments and Methods in Physics Research Section A: Accelerators, Spectrometers, Detectors and Associated Equipment, Volume 595, Issue 1, pp. 200-203, September 2009, doi: 10.1016/j.nima.2008.07.073.

[8] D. Borla Tridon et al., Performance of the Camera of the MAGIC II Telescope, Proceeding of 31st International Cosmic Ray Conference (ICRC 2009), Lodz (Poland), 7 - 15 July 2009, arXiv:0906.5448.

[9] J. Sitarek et al., Analysis techniques and performance of the Domino Ring Sampler version 4 based readout for the MAGIC telescopes, Nuclear Instruments and Methods in Physics Research A: Accelerators, Spectrometers, Detectors and Associated Equipment, Volume 723, pp. 109-120, September 2013, doi: 10.1016/j.nima.2013.05.014.

[10] F. E. Roach and J. L. Gordon, Light of the Night Sky, in Geophysics and Astrophysics Monographs book series (EPIS, volume 8), pp.126, December 1973, doi: 10.1007/978-94-010-2553-9.

[11] R. Paoletti et al., The Trigger System of the MAGIC Telescope, in IEEE Transactions on Nuclear Science, Volume 54, Issue 2, pp. 404-409, April 2007, doi: 10.1016/j.nima.2003.11.083.

[12] R. Paoletti et al., The global trigger system of the magic telescope array, Proceeding of 2008 IEEE Nuclear Science Symposium Conference, Dresden (Germany), 9-25 October 2008, pp. 2781-2783, doi: 10.1109/NSSMIC.2008.4774948.

[13] E. O. Wilhelmi, The Optimization of the MAGIC Telescope for Pulsar Observations, $\mathrm{PhD}$ thesis in Physics, Potchefstroom (South Africa) December 2003.

[14] E. O. Wilhelmi and O. C. De Jager, A New Trigger Principle for Pulsar Observations at Low Energy, Proceeding of Joint European and National Astronomy Meeting (JENAM '04), Granada (Spain), 13 - 17 September 2004.

[15] M. T. Rissi et al., A new trigger provides a lower energy threshold for the MAGIC Cherenkov telescope, Proceeding of Nuclear Science Symposium Conference Record 2008, pp. 1472-1475, October 2008, doi: $10.1109 /$ NSSMIC.2008.4774693.

[16] M. T. Rissi. Detection of pulsed very high energy gamma-rays from the Crab pulsar with the MAGIC telescope using an analog sum trigger, $\mathrm{PhD}$ thesis, Institute for Particle Physics, ETH Zürich, October 2009.

[17] P. Majumdar et al., Monte Carlo simulation for the MAGIC telescope, Proceeding of $29^{t h}$ International Cosmic Ray Conference (ICRC 2005), Pune (India), 3-10 August 2005.

[18] M. Lòpez, Simulations of the MAGIC telescopes with matelsim, Proceeding of $33^{\text {th }}$ International Cosmic Ray Conference (ICRC 2013), Rio de Janeiro (Brazil), 2-9 July 2013.

[19] F. Dazzi, A new stereoscopic Sum-Trigger-II for the MAGIC Telescopes, $\mathrm{PhD}$ thesis in Mathematics \& Physics, University of Udine, Udine (Italy), March 2012.

[20] J. Albert et al., Observation of pulsed $\gamma$-rays above $25 \mathrm{GeV}$ from the Crab Pulsar with MAGIC, in Science, Volume 322, Issue 5905, pp. 1221 - 1224, November 2008, doi: 10.1126/science.1164718.

[21] L. A. Tejedor et al., An Analog Trigger System for Atmospheric Cherenkov Telescope Arrays, in IEEE Transactions on Nuclear Science, Volume 60, Issue 3, pp. 2367-2375, June 2013, doi: 10.1109/TNS.2013.2257852.
[22] B. S. Acharya et al., Introducing the CTA concept, in Astroparticle Physics, Volume 43, March 2013, Pages 3-18, doi: 10.1016/j.astropartphys.2013.01.007.

[23] R. Mirzoyan et al., Recent Highlights of MAGIC, Proceeding of $33^{\text {th }}$ International Cosmic Ray Conference (ICRC 2013), Rio de Janeiro (Brazil), 2-9 July 2013.

[24] D. Paneque et al., The MAGIC of very-high-energy gamma-ray astronomy, Proceeding of Topics in Astroparticle and Underground Physics (TAUP 2019), Toyama (Japan), 8-14 September 2019, Journal of Physics: Conference Series, Volume 1468, 2020.

[25] V. P. Fomin et al., New methods of atmospheric Cherenkov for gammaray astronomy. I. The false source method, Astroparticle Physics, Volume 2, Issue 2, pp. 137-150, May 1994, doi: 10.1016/09276505(94)90036-1.

[26] D. Häfner et al., A Tunable Delay Line for Fast Analog Pulses as Key Element of a New Sum-Trigger for Cherenkov Telescopes, in IEEE Transactions on Nuclear Science, Vol. 59, Issue 2, pp. 289-293, April 2012, doi: 10.1109/TNS.2011.2182619.

[27] R. Mirzoyan et al., On the influence of afterpulsing in PMTs on the trigger threshold of multichannel light detectors in self-trigger mode, in Nuclear Instruments and Methods in Physics Research Section A: Accelerators, Spectrometers, Detectors and Associated Equipment, Volume 387, Issue 1-2, pp. 74-78, March 1997, doi: 10.1016/S01689002(96)00964-3.

[28] R. Mirzoyan, D. Müller et. al., Evaluation of novel PMTs of worldwide best parameters for the CTA project, in Nuclear Instruments and Methods in Physics Research Section A: Accelerators, Spectrometers, Detectors and Associated Equipment, Volume 845, pp. 603-606, 11 February 2017, doi: 10.1016/j.nima.2016.06.080.

[29] D. Tescaro et al., The Readout System of the MAGIC-II Cherenkov Telescope, Proceeding of 31st International Cosmic Ray Conference (ICRC 2009), Lodz (Poland), 7-15 July 2009, arXiv:0907.0466.

[30] R. Zanin and J.Cortina, The Central Control of the MAGIC Telescopes, Proceeding of 31st International Cosmic Ray Conference (ICRC 2009), Lodz (Poland), 7-15 July 2009, arXiv:0907.0946.

[31] J. R. Garcia et al., Status of the new Sum-Trigger system for the MAGIC telescopes, Proceeding of $33^{\text {th }}$ International Cosmic Ray Conference (ICRC 2013), Rio de Janeiro (Brazil), 2-9 July 2013, arXiv:1404.4219.

[32] F. Dazzi et al., Performance studies of the new stereoscopic SumTrigger-II of MAGIC after one year of operation, Proceeding of $34^{\text {th }}$ International Cosmic Ray Conference (ICRC 2015), The Hague (Netherlands), 30 July - 6 August 2015, arXiv:1508.05255.

[33] G. Ceribella et al., MAGIC studies of the Crab Pulsar and Nebula spectra for energies above $20 \mathrm{GeV}$, Proceeding of $36^{\text {th }}$ International Cosmic Ray Conference (ICRC 2019), Madison (USA), 24 July - 1 August 2019 , PoS(ICRC2019)645.

[34] M. López et al., Detection of the Geminga pulsar with the MAGIC telescopes, Proceeding of $36^{\text {th }}$ International Cosmic Ray Conference (ICRC 2019), Madison (USA), 24 July - 1 August 2019, PoS(ICRC2019)728.

[35] V. A. Acciari et al., Detection of the Geminga pulsar with MAGIC hints at a power-law tail emission beyond $15 \mathrm{GeV}$, in Astronomy and Astrophysics, Vol. 643, pp. L14, November 2020, doi: 10.1051/0004$6361 / 202039131$. 